\title{
Early Weak Fault Diagnosis of Rolling Bearing Based on Multilayer Reconstruction Filter
}

\author{
Quanfu Li, ${ }^{1,2}$ Yuxuan Zhou $\mathbb{D}^{3},{ }^{3}$ Gang Tang $\mathbb{D}^{3},{ }^{3}$ Chunlin Xin $\mathbb{D D}^{3}$ and Tao Zhang ${ }^{4}$ \\ ${ }^{1}$ Southwest Jiaotong University, Chengdu 611756, China \\ ${ }^{2}$ Shenhua Railway Equipment Co. Ltd., Beijing 100120, China \\ ${ }^{3}$ Beijing University of Chemical Technology, Beijing 100029, China \\ ${ }^{4}$ AECC Hunan Aviation Powerplant Research Institute, AECC Key Laboratory of Aero-engine Vibration Technology, \\ Zhuzhou 412002, Hunan, China
}

Correspondence should be addressed to Gang Tang; tanggang@mail.buct.edu.cn

Received 11 December 2020; Revised 5 January 2021; Accepted 21 January 2021; Published 2 March 2021

Academic Editor: Jinde Zheng

Copyright (c) 2021 Quanfu Li et al. This is an open access article distributed under the Creative Commons Attribution License, which permits unrestricted use, distribution, and reproduction in any medium, provided the original work is properly cited.

The early weak fault characteristics of rolling bearings are extremely weak and are easily overwhelmed by other noises. In order to effectively extract the characteristics of the early weak faults of the rolling bearings and draw on the multilayer wavelet decomposition idea, a method for diagnosing the early weak faults of the rolling bearing based on the multilayer reconstruction filter is proposed. As we all know, empirical wavelet transform (EWT) makes full use of wavelet filter bank, and variational mode decomposition (VMD) uses Wiener filter bank. This paper fully combines the advantages of the above two methods, adaptively determines the number of modes through empirical wavelet decomposition and divides the original signal, extracts the frequency band that contains the fault characteristic information, and effectively eliminates noise interference. These steps are repeated until the optimal component of the condition is obtained. In the output layer, the weak fault impact components are further separated by the strong filtering and signal decomposition capability of VMD. The advantages of the proposed method are proved by the experiment of weak fault of rolling bearing and the accelerated failure experiment of full life. The proposed method has the advantages of reducing noise influence and adaptive estimation of decomposed modes, which can be applied more efficiently in practice.

\section{Introduction}

Timely and effective identification of early weak faults for rolling bearings has an important significance to ensure the safety of equipment operation [1]. The small impact of early weak faults leads to weak fault characteristics. At the same time, the signal usually contains a large number of noise interferences caused by the mutual impact of other components, which brings about great challenges to relevant studies $[2,3]$.

Data-driven fault diagnosis methods for rolling bearings have been the most widely studied, most of which are based on vibration signals and acoustic signals, which are essentially the same, but each has its own advantages and disadvantages [4]. The advantages of vibration signal used in fault diagnosis are as follows: the cost of the sensor is relatively low, the vibration signal is easy to be measured, and the measured vibration signal can contain more fault information. Its disadvantages are as follows: this is a kind of contact measurement, and the sensor needs to be installed in the position close to the workpiece. The advantages of using acoustic signals for fault diagnosis are as follows: the requirements for the installation position are not very strict, they do not need to be attached to the workpiece under test, and the measurement cost is relatively low. The disadvantage is that the measured acoustic signal may contain sound other than the target workpiece, so the SNR is relatively small [5]. Adam Glowacz used acoustic signals for early bearing and stator of the single-phase induction motor 
fault diagnosis [6]. Parey and Singh proposed a continuous wavelet transform and adaptive neural fuzzy reasoning system to process the acoustic signal of gearbox and complete fault diagnosis [7]. Adam Glowacz proposed a fault diagnosis method of single-phase motor based on acoustic signal [5]. Zhang and Stewart proposed a deep graph convolutional network for roller bearings based on acoustic signal [8]. Liu and Pei achieved fault detection on belt conveyor idlers by processing acoustic signals using machine learning method [9]. In the application of vibration signals for fault diagnosis, Huang et al. extracted frequency band entropy from vibration signals for fault diagnosis [10]. Wang et al. used timefrequency curves of vibration signals for fault detection under variable speed [11].

In the past decades, researchers have proposed many methods for the rolling bearing fault diagnosis, among which the adaptive signal decomposition method is quite popular [12]. Common adaptive decomposition methods include empirical mode decomposition (EMD), EWT, and VMD [13]. EMD has been widely used in various fields $[14,15]$. Due to its difficulty in mathematical modeling, sensitivity to noise, and endpoint effect, scholars have developed a lot of improved EMD, but it still cannot achieve ideal results [16]. In order to overcome the limitations of EMD, Jerome Gilles and Konstantin Dragomiretskiy proposed EWT [17] and VMD [18], respectively. The EWT constructs an adaptive empirical wavelet filter bank and the variational modal decomposition fully borrows the Wiener filter. Both of them have been fully studied in the field of rolling bearing fault diagnosis.

Some researchers made a detailed comparative study on EWT and EMD and concluded that EWT has better performance than EEMD and EMD in model estimation [19]. EWT has been widely used in the fields of medical signal analysis, seismic signal analysis, meteorological prediction, and fault diagnosis [20-23]. In the field of fault diagnosis, some papers have improved EWT from the aspects of spectrum segmentation, so that it can be better applied to practical engineering problems [17, 24]. VMD obtains all modalities from the signal at the same time through a joint optimization scheme, so it has higher resolution [25]. Compared with EWT, this method has been widely used. A large number of scholars have also improved the VMD. Some scholars use the dimensionality-increasing feature of VMD to apply it to underdetermined blind source separation [26]. Jiang et al. [27] proposed a VMD decomposition strategy from rough to fine, which was well applied to the fault diagnosis of rotating equipment. Many scholars have also studied the parameter optimization of VMD to improve its adaptive ability [28, 29]. Xu et al. [30] proposed a method based on VMD which can reduce noise in the propagation path. Although EWT and VMD have been widely studied in the field of rolling bearing fault diagnosis, they still have some room for improvement.

Due to extremely weak fault characteristics and large amount of noise interference in vibration signal and acoustic signal, it is a great challenge to extract fault characteristic information effectively. Some scholars have combined the mode decomposition method with other methods. For example, Fan [2] combined with the advantages of EMD to improve VMD and achieved certain results.

This method utilizes EWT's great capability of mode number estimation [17] and its excellent filtering capability of the empirical wavelet filter bank and combines the kurtosis criterion to effectively extract the optimal mode from the original signal. Multilayer reconstruction filter can remove most of the noise components and highlight the fault impact components. However, due to the weak fault features, it is usually not possible to directly extract the fault characteristics from the denoised signal. By virtue of the great band-pass filtering capability and signal decomposition capability of VMD, it is used as the output layer to reconstruct and filter the signal after noise reduction. Because the multilayer reconstruction filter reduces the interference of redundant information to the VMD, the fault feature information can be separated and extracted better, and the early weak fault diagnosis of rolling bearing can be realized.

The structure of this paper is as follows: Section 2 introduces the theoretical basis of the method and introduces the process of the proposed method; Section 3 verifies the proposed method by using the acoustic signal of rolling bearing fault experiment and the vibration signal of accelerated fatigue experiment, respectively. The results and discussion are given in Section 4.

\section{Methodology}

2.1. Empirical Wavelet Transform. The EWT divides the Fourier spectrum of a signal and constructs a wavelet filter bank to extract the intrinsic modes and can be divided into several main components.

Assuming that the Fourier support $[0, \pi]$ is segmented into contiguous $N$ segments, a total number of $N+1$ boundaries are needed. According to the local maximum values, the Fourier spectrum of a signal is divided into $N$ segments. Denote $\omega_{n}$ to be the limits between each segment (where $\omega_{0}=0$ and $\omega_{n}=\pi$ ). Each segment is represented as $\Lambda_{n}=\left[\omega_{n-1}, \omega_{n}\right]$. A transition phase $T_{n}$ of width $2 \tau_{n}$ is then defined, as shown in Figure 1.

The empirical wavelets are defined as band-pass filters on each $\Lambda_{n}$. The empirical scale function $\hat{\phi}(\omega)$ and empirical wavelet function $\hat{\psi}(\omega)$ can be calculated by the two following equations, respectively: 


$$
\begin{aligned}
& \hat{\phi}(\omega)= \begin{cases}1, & \text { if }|\omega| \leq(1-\gamma) \omega_{n}, \\
\cos \left[\frac{\pi}{2}\left(\frac{1}{2 \gamma \omega_{n}}\left(|\omega|-(1-\gamma) \omega_{n}\right)\right)\right], & \text { if }(1-\gamma) \omega_{n} \leq|\omega| \leq(1+\gamma) \omega_{n}, \\
0, & \text { otherwise, }\end{cases} \\
& \hat{\psi}(\omega)= \begin{cases}1, & \text { if }(1+\gamma) \omega_{n} \leq|\omega| \leq(1-\gamma) \omega_{n+1}, \\
\cos \left[\frac{\pi}{2} \beta\left(\frac{1}{2 \gamma \omega_{n+1}}\left(|\omega|-(1-\gamma) \omega_{n+1}\right)\right)\right], & \text { if }(1-\gamma) \omega_{n+1} \leq|\omega| \leq(1+\gamma) \omega_{n+1}, \\
\sin \left[\frac{\pi}{2} \beta\left(\frac{1}{2 \gamma \omega_{n+1}}\left(|\omega|-(1-\gamma) \omega_{n}\right)\right)\right], & \text { if }(1-\gamma) \omega_{n+1} \leq|\omega| \leq(1+\gamma) \omega_{n+1}, \\
0, & \text { otherwise. }\end{cases}
\end{aligned}
$$

The ratio $\gamma$ in equations (1) and (2) is restricted to a small value as $0 \leq \gamma \leq \min _{n}\left[\left(\omega_{n+1}-\omega_{n}\right) /\left(\omega_{n+1}+\omega_{n}\right)\right]$ to ensure the empirical scaling function and the empirical wavelets are a tight frame of $\omega_{n}: \tau_{n}=\gamma \omega_{n}, 0<\gamma<1$. The function $\beta(x)$ is an arbitrary $C^{k}([0,1])$ function, and $\beta(x)$ is defined as follows:

$$
\beta(x)=x^{4}\left(35-84 x+70 x^{2}+20 x^{3}\right) .
$$

The approximation coefficients $w_{f}^{\varepsilon}(0, t)$ and the detail coefficients $w_{f}^{\varepsilon}(n, t)$ can be calculated by the two following equations, respectively:

$$
\begin{aligned}
w_{f}^{\varepsilon}(0, t) & =f, \\
\phi_{1} & =\int f(\tau) \overline{\phi_{1}(\tau-t)} \mathrm{d} \tau=\left(\hat{f}(\omega) \overline{\hat{\phi}_{1}(\omega)}\right), \\
w_{f}^{\varepsilon}(n, t) & =f, \\
\psi_{n} & =\int f(\tau) \overline{\psi_{n}(\tau-t)} \mathrm{d} \tau=\left(\hat{f}(\omega) \overline{\hat{\psi}_{n}(\omega)}\right)^{\vee},
\end{aligned}
$$

where $\hat{\phi}_{1}(\omega)$ and $\hat{\psi}_{n}(\omega)$ are defined by equations (1) and (2), respectively.

The inverse empirical wavelet transformation is carried out by the following equation:

$$
\begin{aligned}
f(t) & =w_{f}^{\varepsilon}(0, t) * \phi_{1}(t)+\sum_{n=1}^{N} w_{f}^{\varepsilon}(n, t) * \psi_{n}(t), \\
& =\left(\hat{w}_{f}^{\varepsilon}(0, \omega) * \hat{\phi}_{1}(\omega)+\sum_{n=1}^{\hat{N}} \hat{w}_{f}^{\varepsilon}(n, \omega) * \hat{\psi}_{n}(\omega)\right)^{\vee} .
\end{aligned}
$$

2.2. Variational Mode Decomposition. Variational modal decomposition is an adaptive time-frequency analysis tool, which decomposes the signal into multiple BLIMFs (band limited intrinsic mode function) through iterative solution.
The detailed steps are described below.

First, it is assumed that the signal $x(t)$ can be decomposed into a finite number of inherent modes, each of which has a different center frequency and $\omega_{k}$, a finite bandwidth.

The specific formula of the unilateral spectrum obtained by Fourier transform is as follows:

$$
\left(\delta(t)+\frac{j}{\pi t}\right) * u_{k}(t)
$$

In the above formula, $\delta(t)$ stands for Dirichlet function, and $*$ stands for convolution.

The spectrum of each component is obtained by the following equation:

$$
\left[\left(\delta(t)+\frac{j}{\pi t}\right) * u_{k}(t)\right] e^{j \omega_{k} t}
$$

The variational constraint mode function of VMD is as follows:

$$
\begin{aligned}
& \min _{\left\{u_{k}\right\},\left\{w_{k}\right\}}\left\{\sum_{k}\left\|\partial_{t}\left[\left(\delta(t)+\frac{j}{\pi t}\right) * u_{k}(t)\right] e^{-j w_{k} t}\right\|_{2}^{2}\right\}, \\
& \text { s.t. } \quad \sum_{k} u_{k}=x .
\end{aligned}
$$

In the above formula, $\left\{u_{k}\right\}=\left\{u_{1}, \ldots, u_{K}\right\}$ represents the $\mathrm{K}$ components obtained after decomposition; $\left\{\omega_{k}\right\}=\left\{\omega_{1}, \ldots, \omega_{K}\right\}$ represents the center frequency corresponding to each component.

In order to obtain the optimal solution of the above variational constraint model, the VMD algorithm introduces the Lagrangian method, including the secondary punishment factor $\alpha$ and the Lagrangian multiplier $\lambda$. The formula is as follows: 


$$
L=\alpha \sum_{k}\left\|\partial_{t}\left[\left(\delta(t)+\frac{j}{\pi t}\right) * u_{k}(t)\right] e^{-j w_{k} t}\right\|_{2}^{2}+\left\|x(t)-\sum_{k} u_{k}(t)\right\|_{2}^{2}+\lambda(t), x(t)-\sum_{k} u_{k}(t) .
$$

Iteratively update $\left\{u_{k}\right\},\left\{w_{k}\right\}$, and finally obtain the saddle point of Lagrange equation, which is the optimal solution.

The specific steps of the VMD procedure are shown in Figure 2.

\subsection{Proposed Method: Multilayer Reconstruction Filter.} The VMD method has a reliable theoretical basis, with bandpass filtering capability similar to wavelet packet decomposition and excellent signal decomposition capability. However, weak fault pulses and strong background noise can seriously affect the accuracy of diagnosis. Therefore, drawing on the idea of multilayer wavelet decomposition, this paper introduces a powerful signal processing tool, EWT, as a preprocessing. EWT has an outstanding ability to detect the number of modes. This method divides the signal in the Fourier domain by looking for the local maximum and divides the signal into multiple frequency bands adaptively. By constructing an empirical wavelet filter bank with excellent performance, each frequency band is reconstructed and filtered to effectively eliminate the interference of nonimpulsive noise. As a fourth-order high-order statistic equation (11), kurtosis is very sensitive to early failures. The correlation coefficient can choose the most containing fault information frequency band. Therefore, with the help of the above two coefficients, a relatively optimal component can be well selected. Using this component as the input signal of the next layer, perform the above process again until the set number of decomposition layers is reached to obtain the optimal mode.

$$
K=\frac{1}{N} \sum_{i=1}^{N}\left[\frac{x_{i}(t)-\mu}{\sigma}\right]^{4}
$$

In the above formula, $N$ is the length of the signal, $x_{i}$ is the $i$-th EWT component, and $\mu$ and $\sigma$ are the mean value and standard deviation of the signal, respectively.

Compared with the original signal, the selected optimal component has very low noise interference and no excessive redundant information. Taking this component as the input of the output layer, it can better exert the powerful signal filtering and decomposition ability of VMD, effectively decompose the fault pulse information, and realize accurate fault diagnosis. The flow chart of the proposed method is shown in Figure 3.

\section{Experiment and Results}

The method proposed in this paper aims at the early weak faults of rolling bearings with extremely weak fault characteristics and extremely high background noise. This chapter uses two related experiments for analysis. In experiment 1 , weak faults were made in the inner and outer rings of the bearing ring by using laser cutting technology, and acoustic signals were collected by microphone sensor. The acoustic signals collected by this experiment met the premise of weak fault characteristics and great background noise. In experiment 2 , the bearing accelerated degradation experiment of NSF I/UCR Intelligent Maintenance Systems was adopted. The experiment collected data on the entire life cycle of the bearing, which truly reflects the entire stage of failure from early initiation and development to destruction and shutdown, which is well in line with the needs of experimental research. Two experiments are presented below.

\subsection{Simulation Experiment Analysis of Early Fault of Rolling Bearing}

3.1.1. Experiment Design. In this paper, a rolling bearing fault test rig is designed, as shown in Figure 4. The type of bearing is NSK NU205EW, whose parameters are shown in Table 1. Laser cutting method is adopted to cut through $0.1 \mathrm{~mm}$ wide and $0.05 \mathrm{~mm}$ deep grooves in the inner and outer rings of bearing. Acquisition card was used to record the experimental data, MPA416 microphone was used to collect the acoustic signal, the sensor was installed in front of the faulty bearing, the distance was $30 \mathrm{~mm}$, and the photoelectric encoder was used to record the speed. Experimental bench is with rated power of $1.5 \mathrm{~kW}$, rated voltage of $380 \mathrm{~V}$, rated frequency of $50 \mathrm{~Hz}$, rated current of $3.4 \mathrm{~A}$, rated speed of $2840 \mathrm{r} / \mathrm{min}$ of three-phase asynchronous motor drive, transverse load of $2 \mathrm{kN}$, sampling frequency of $20 \mathrm{kHz}$, and speed of $1309.2 \mathrm{r} / \mathrm{min}(f=21.82 \mathrm{~Hz})$. The inner and outer ring fault frequencies of the bearings are $169.1 \mathrm{~Hz}$ and $114.6 \mathrm{~Hz}$, respectively. Equations (12)-(14), respectively, give the calculation formula of fault characteristic frequency of bearing's inner ring, outer ring, and rolling element.where $f_{I}, f_{O}, f_{B}$, respectively, represent the characteristic frequency of inner ring fault, the characteristic frequency of outer ring fault, and the characteristic frequency of rolling element fault (unit: $\mathrm{Hz}$ ); $r$ is the speed of rotation of the bearing (unit: $(r / \mathrm{min})) ; n$ denotes ball number; $d$ denotes the diameter of the rolling element (unit: $\mathrm{mm}$ ); $D$ denotes bearing pitch diameter (unit: $\mathrm{mm}$ ); and $\alpha$ denotes contact Angle (unit: ○).

$$
\begin{aligned}
& f_{I}=\frac{n}{2} \frac{r}{60}\left(1+\left(\frac{d}{D} \cos \alpha\right)\right), \\
& f_{O}=\frac{n}{2} \frac{r}{60}\left(1-\left(\frac{d}{D} \cos \alpha\right)\right), \\
& f_{B}=\frac{n}{2} \frac{r}{60} \frac{D}{d}\left(1-\left(\frac{d}{D} \cos \alpha\right)^{2}\right),
\end{aligned}
$$

3.1.2. Experimental Results and Analysis. First, the time domain waveform and Hilbert envelope spectrum of the original signal were made, as shown in Figures 5 and 6, 


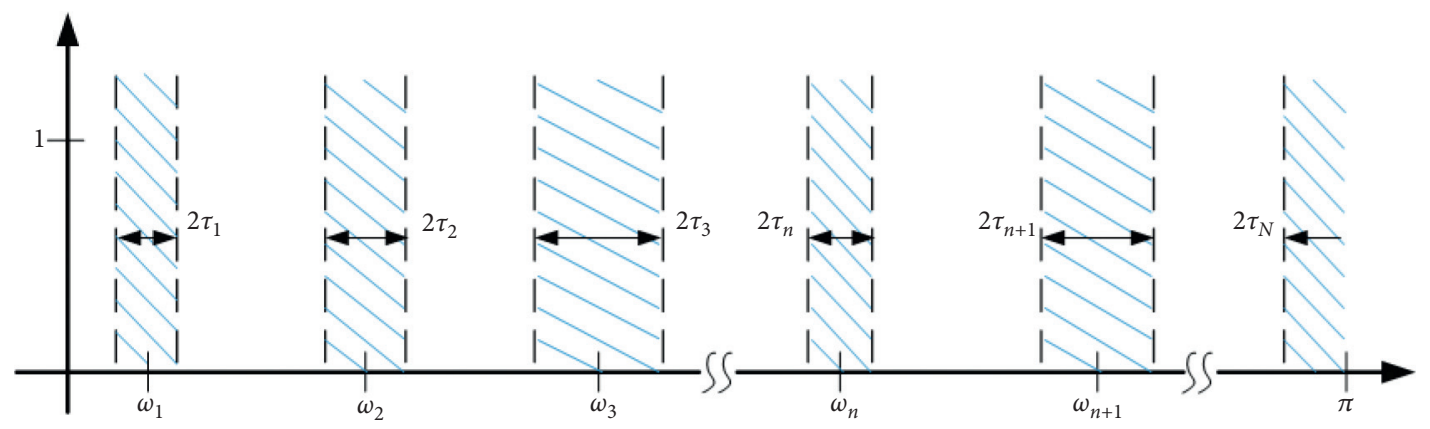

Figure 1: Partitioning of the Fourier axis.

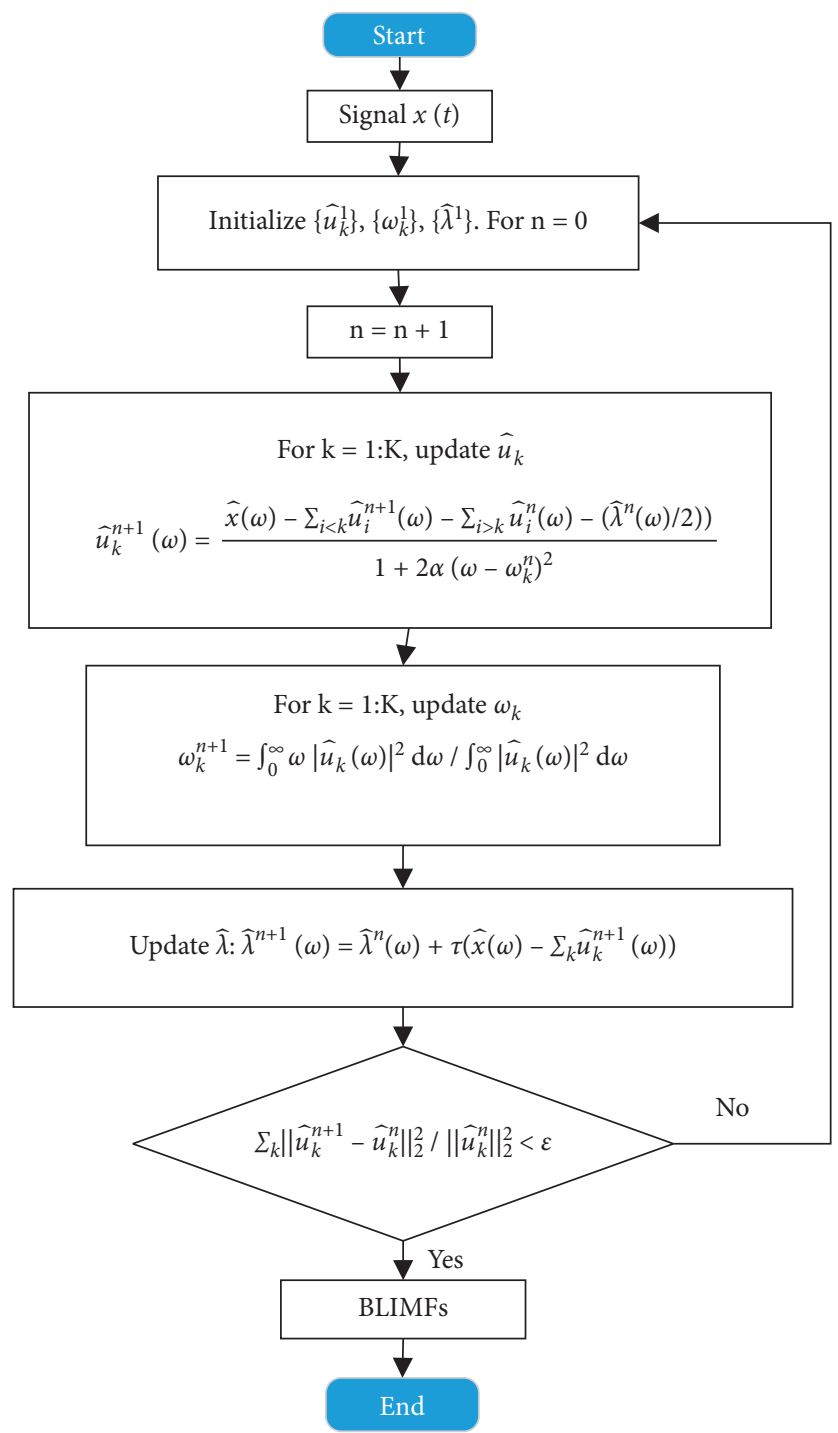

FIgURE 2: Variational mode decomposition algorithm flow.

respectively. The time domain diagram shows that the signal amplitude is low and unobvious periodic impact is found. Further observation of the signal's Hilbert envelope spectrum revealed a large number of irrelevant frequencies, especially in the low frequency region, indicating that the original signal contained a large number of noise interferences. From the spectrogram, only the inner ring fault characteristic frequency with less obvious amplitude can be found, but the inner ring fault characteristic frequency cannot be found, which is enough to prove the premise of 


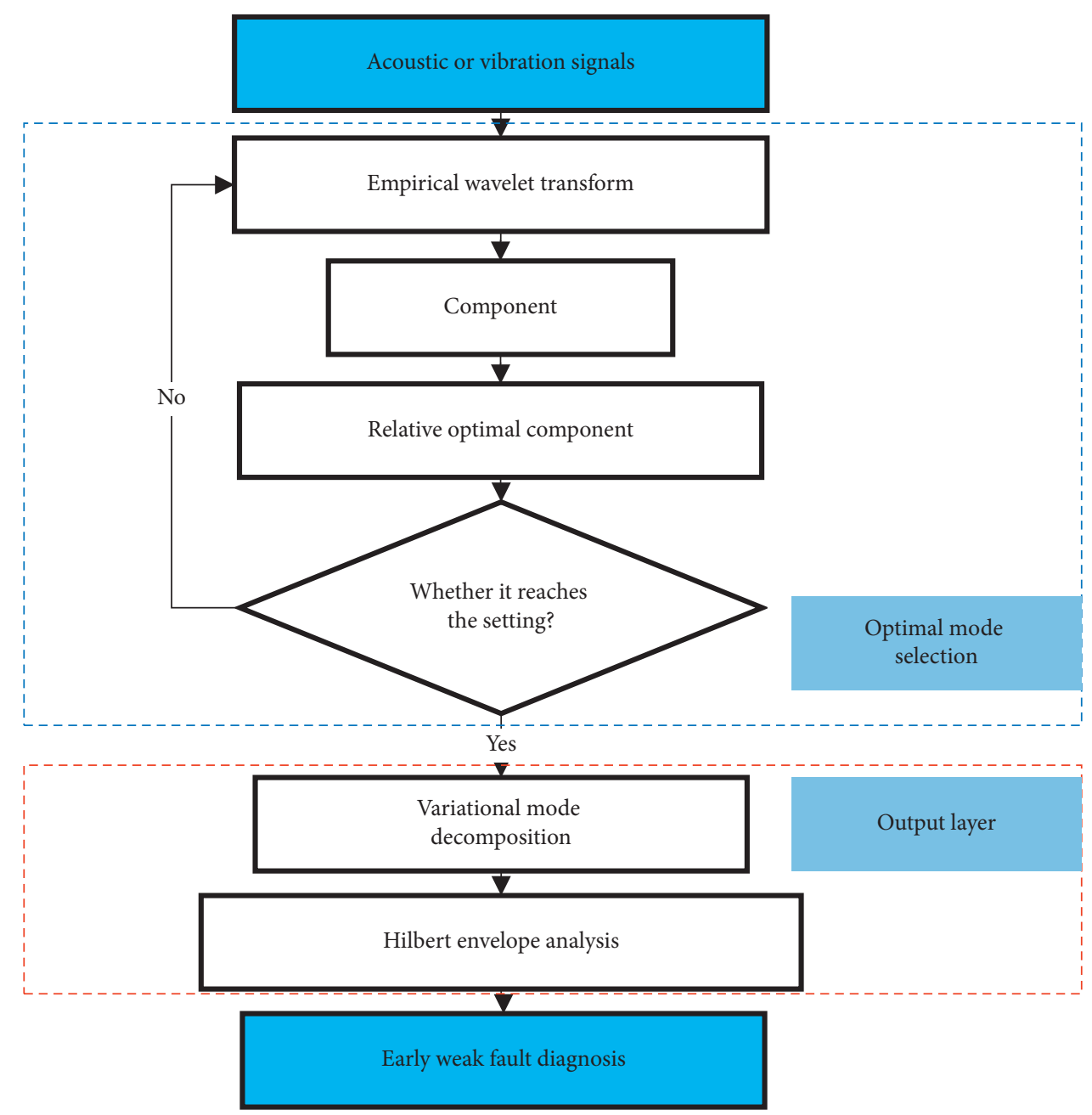

Figure 3: The flow chart of the proposed method.

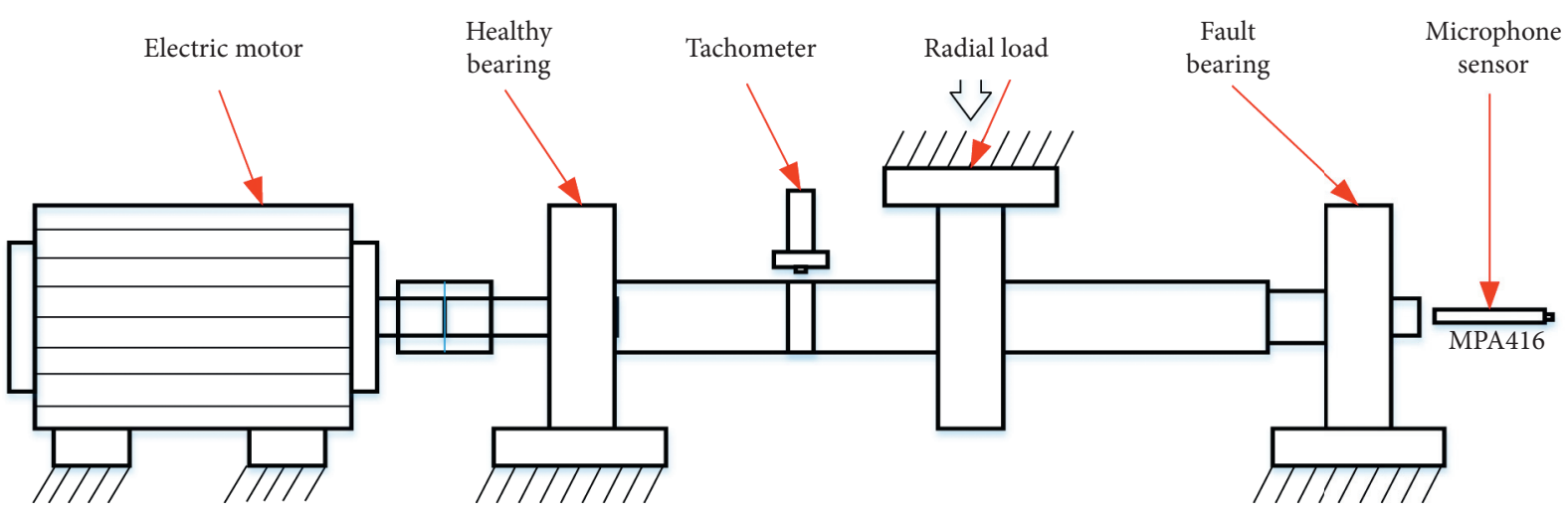

FIgURE 4: Rolling bearing fault test rig.

TABLE 1: Basic parameters of NSK NU205EW bearing.

\begin{tabular}{lccc}
\hline Bearing pitch diameter & Roller diameter & Ball number & Contact angle \\
\hline 39 & 7.5 & 12 & $0^{\circ}$ \\
\hline
\end{tabular}




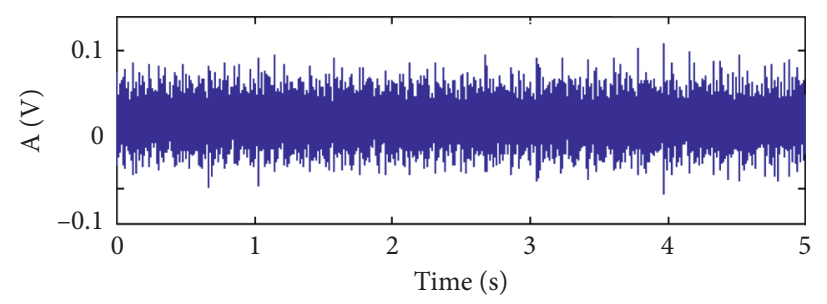

FiguRE 5: Time domain waveform of the original signal of experiment 1.

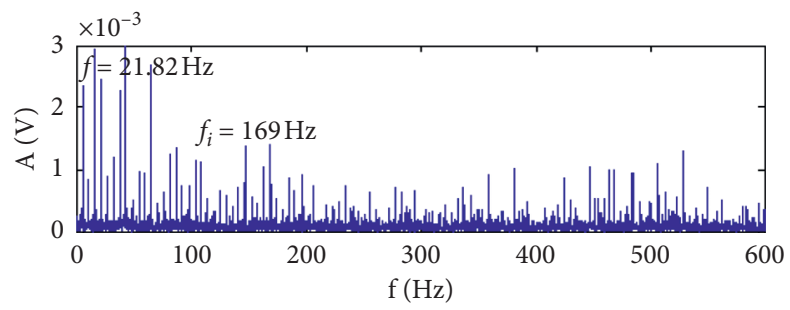

FIgURE 6: The original signal envelope of experiment 1.

weak fault characteristics and large noise interference. The following describes the specific process of signal processing using the method proposed in this paper.

The first reconstruction filter is performed. EWT is used to decompose the signal, and Figure 7 is the boundary detection graph. From the figure, EWT can detect 4 boundaries in the Fourier spectrum and divide the signal into 5 parts. By constructing an empirical wavelet filter bank, band-pass filtering is carried out on these 5 parts, and 5 components are finally obtained. Figure 8 shows the envelope spectra corresponding to the 5 components from top to bottom, and the corresponding correlation coefficients and kurtosis are given in Table 2.

According to the correlation coefficient, we can get that component 5 contains the richest information, which can also be seen from its corresponding envelope spectrum. At the same time, its envelope spectrum can clearly find the inner ring fault characteristic frequency and its multiplier, but it still cannot find the outer ring fault characteristic frequency. It also proves that primary filtering is insufficient for this early weak fault diagnosis. Meanwhile, the kurtosis of component 5 also reflects that it contains some fault pulse information.

Component 2 has the highest kurtosis, and the corresponding envelope spectrum can also find the inner ring fault characteristic frequency, rotation frequency, and its doubling frequency more clearly, so it can be judged that component 2 contains valid fault pulse information.

Based on the above two points, we choose the combination of components 2 and 5 as the optimal mode component. Through the above steps, part of the interference noise in the original signal has been removed, and a part of the fault features have been highlighted, laying a good foundation for the next processing.

Then input the reconstructed signal to the output layer after filtering, and set $K=5$ and $\alpha=2000$ for VMD [31].
Figure 9 shows the envelope spectrum of the BLIMF component obtained after the decomposition of VMD.

Careful analysis of the envelope spectra of the above 5 components shows that, in component 5 , the fundamental frequency and frequency multiplication of the rotation frequency, the characteristic frequency of the inner ring fault, the characteristic frequency of the outer ring fault, and the theoretical calculation can be clearly found. There is almost no difference from the theoretically calculated frequency. Corresponding side bands are also found near the inner ring fault frequency, and the entire frequency spectrum contains almost no other noise interferences. The experimental results fully reflect the accuracy of the proposed method for early weak fault diagnosis.

\subsection{Experimental Analysis of Full-Life Acceleration of Rolling Bearings}

3.2.1. Experiment Design. Experiment 2 adopts the NSF I/ UCR intelligent maintenance system center bearing life accelerated damage experiment data, which is used by many scholars for related research and is an internationally recognized data set. This data covers the whole process from the initiation of the fault to the failure, so it can reflect the damage characteristics of the actual early failure of the bearing and better reflect the effectiveness of the proposed method.

The motor transmits power to the shaft through a belt. Four bearings are mounted on the shaft. At the same time, a constant radial load of $6000 \mathrm{lbs}$ is applied to the shaft. The speed is $2000 \mathrm{r} / \mathrm{min}$, the bearing type is Rexnord ZA-2115 double row bearing, and the basic parameters are shown in Table 3. During operation, the bearing will be worn under heavy load and produce metal debris. A magnetic screw plug is installed on the test bench to collect metal debris. When 


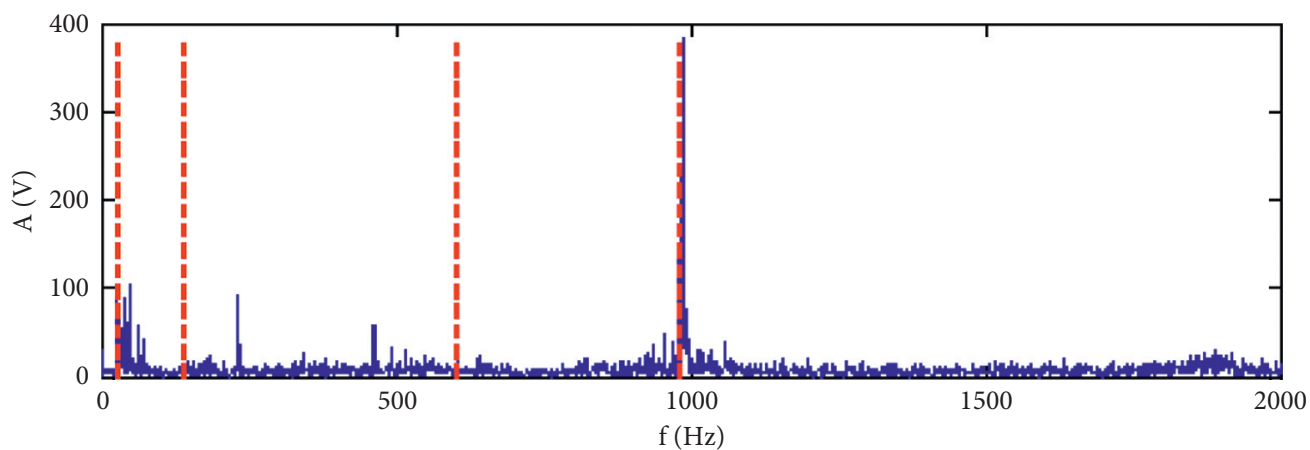

FIgURE 7: EWT boundary detection diagram of experiment 1.
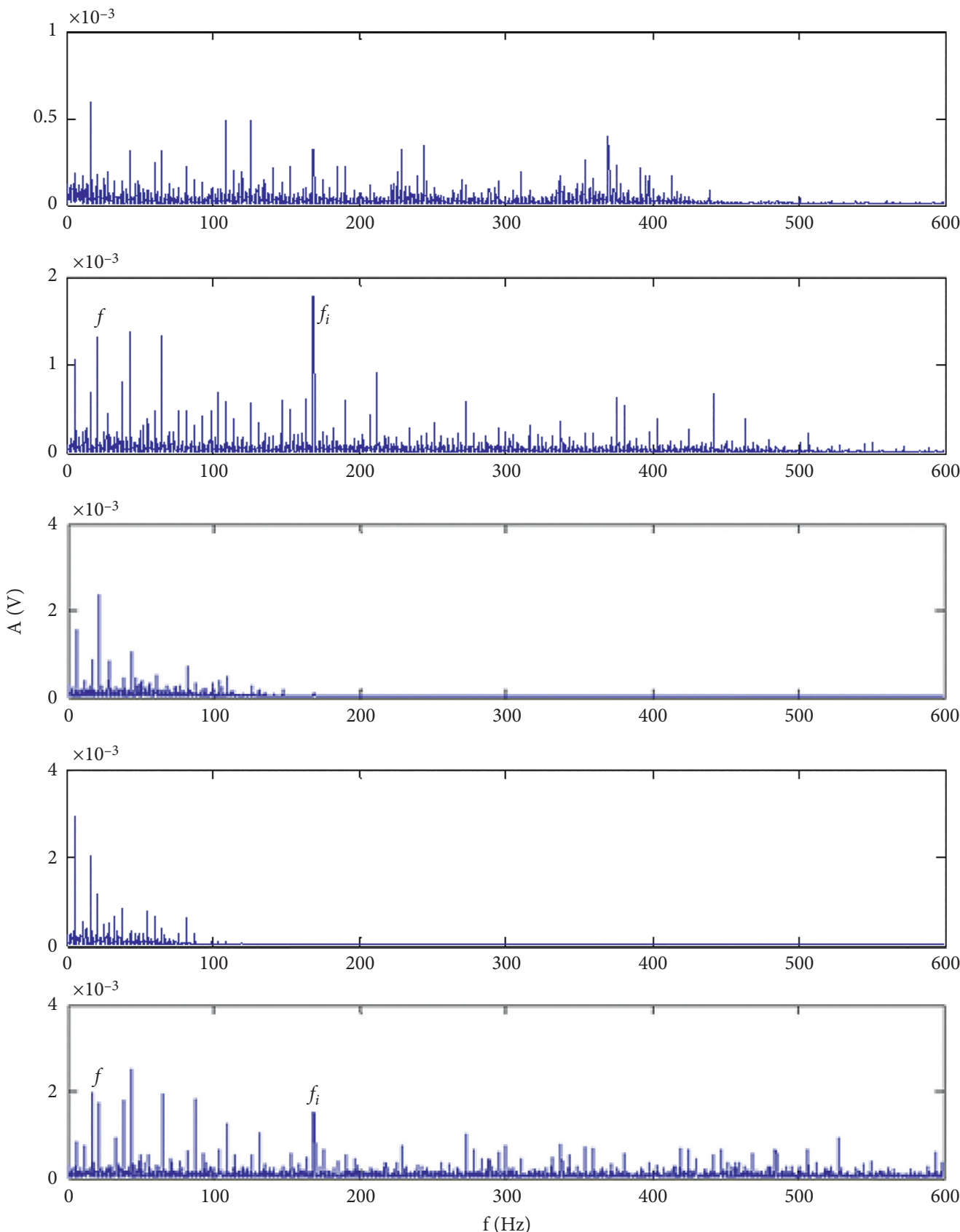

FIgURE 8: EWT component envelope spectra of experiment 1. 
TABLE 2: Correlation coefficient and kurtosis of EWT component of experiment 1.

\begin{tabular}{lcc}
\hline & Correlation coefficient & Kurtosis \\
\hline Component 1 & 0.19 & 2.93 \\
Component 2 & 0.36 & 4.54 \\
Component 3 & 0.33 & 3.21 \\
Component 4 & 0.41 & 3.51 \\
Component 5 & 0.83 & 3.84 \\
\hline
\end{tabular}
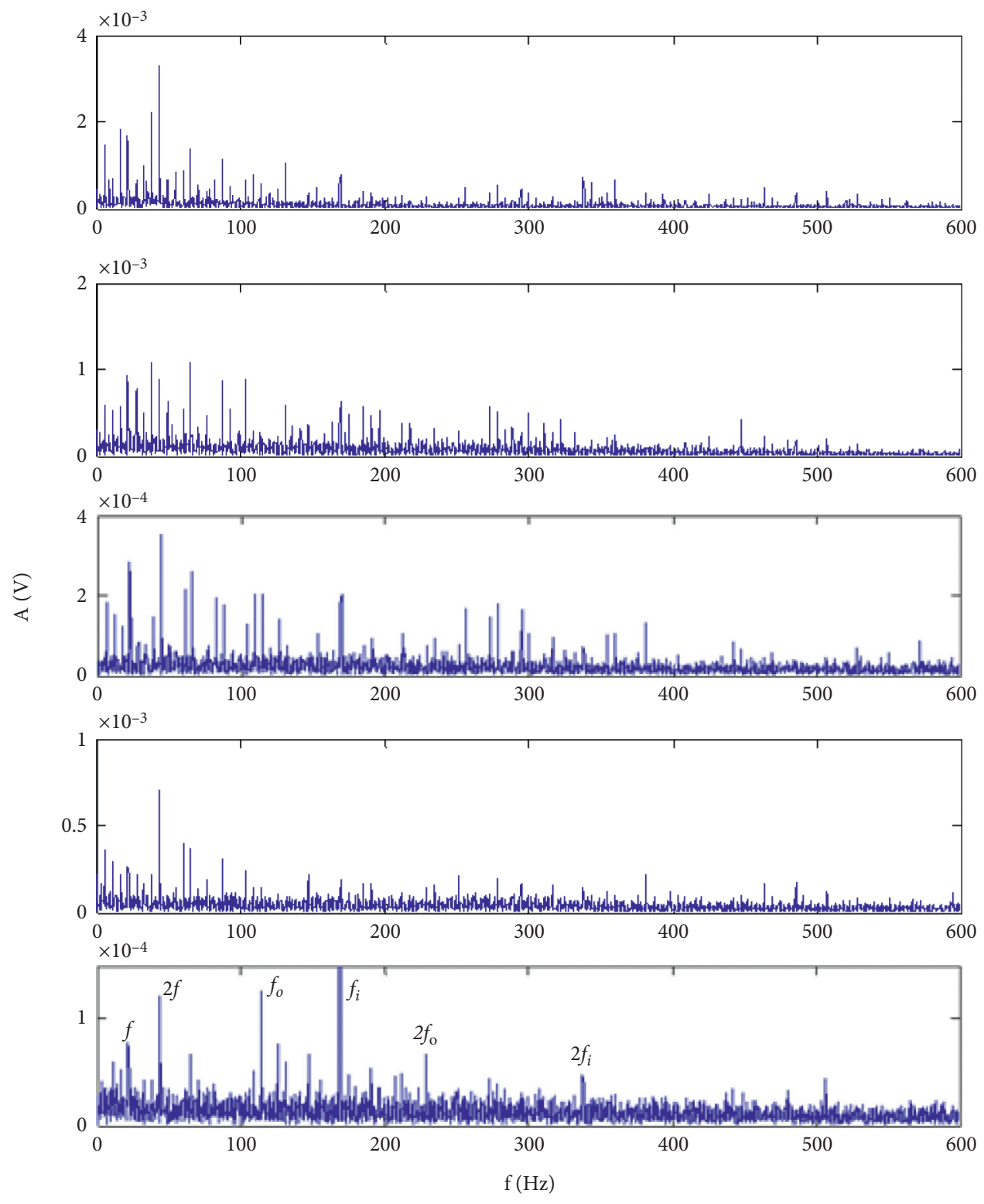

FIGURE 9: VMD component envelope spectrum of experiment 1.

the accumulation of metal debris exceeds a certain level, the electrical switch is automatically controlled to stop.

The experiment consists of three sets of data sets, and each set of data sets contains the complete degradation process data from the beginning of the bearing to the damage. In this part, the second data set is adopted. The sampling frequency is set at $20 \mathrm{kHz}$ and the sampling time is 1s. A total of 20480 acceleration data points are collected in each sampling time, with a sampling interval of $10 \mathrm{~min}$.

3.2.2. Experimental Results and Analysis. The rolling bearing fault test rig is shown in Figure 10. The change curve of the root mean square value of each collection point of the 
TABLE 3: Basic parameters of Rexnord ZA-2115 double-row bearing.

\begin{tabular}{lccc}
\hline Bearing pitch diameter $(\mathrm{mm})$ & Roller diameter $(\mathrm{mm})$ & Ball number & Contact angle \\
\hline 71.5 & 8.4 & 16 & $15.17^{\circ}$ \\
\hline
\end{tabular}

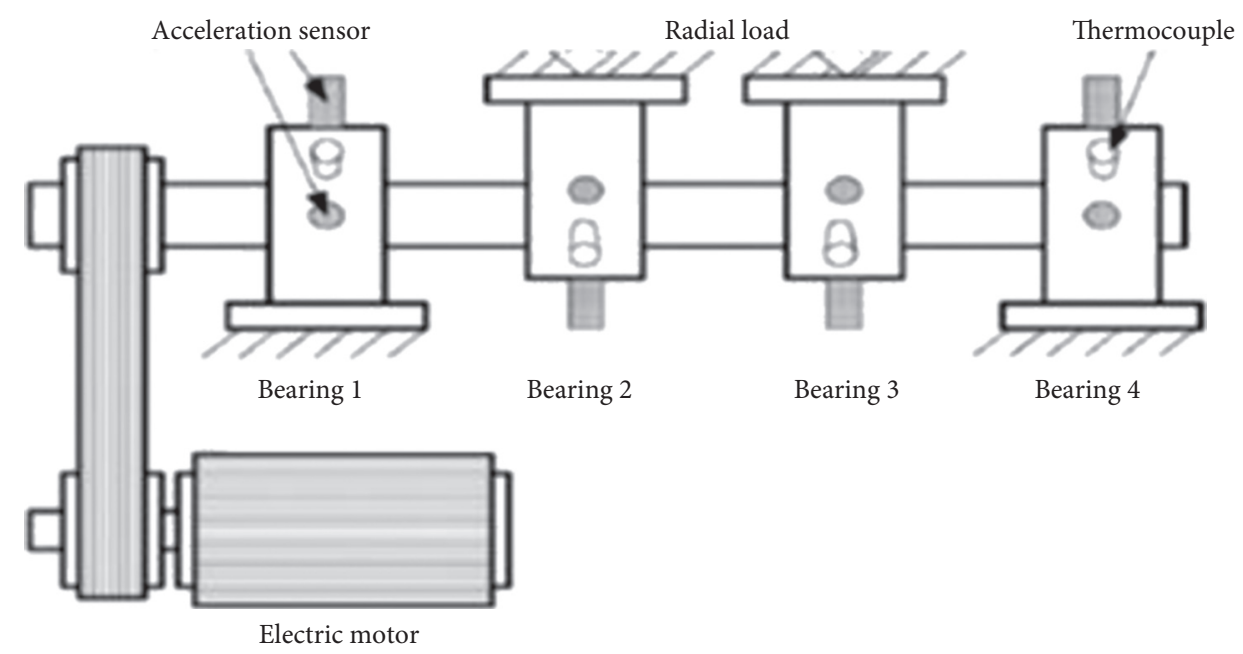

FIgURE 10: Rolling bearing accelerated degradation rig.

whole life data is shown in Figure 11. Since the RMS of the fault state and the normal state will be different under normal circumstances, most scholars regard the mutation points at $\mathrm{T} 1$ (about $5320 \mathrm{~min}$ ) and $\mathrm{T} 2$ (about $6460 \mathrm{~min}$ ) as the fault occurrence point. However, through the research in this article, it is found that the failure may have occurred earlier.

First of all, it can be found in Figure 11 that the signal RMS has not changed significantly around the 4520th minute. Analyzing its time domain waveform and envelope spectrum in Figures 12 and 13, no obvious periodic shock nor obvious fault characteristic information appears, which indicates that even if a fault occurs at this point, it is an extremely weak early fault, or no fault has occurred.

The experimental data were analyzed using the proposed method. The result of EWT boundary detection is shown in Figure 14. First, the signal is decomposed into 5 components in the first layer. Figure 15 shows the envelope spectrum of each component. In the envelope spectrum of these 5 components, no relevant fault characteristic information can be found, and a large number of interference frequencies are included. In component 3 , a frequency component similar to the fault frequency of the outer ring was found, but it was not accurate (the fault frequency of the outer ring was $230.7 \mathrm{~Hz}$, while the frequency was $232.5 \mathrm{~Hz}$ ). Therefore, a layer of filter analysis could not effectively identify whether the bearing fault occurred and the type of fault. This also indicates that the actual fault at 4520 minutes in experiment 2 is weaker and more difficult to identify than the simulated fault in experiment 1 .

The correlation coefficients and kurtosis corresponding to each component are given in Table 4. Through the comprehensive analysis of correlation coefficient, kurtosis, and envelope spectrum, component 5 is selected as the reconstructed signal after a layer filtering. Component 5 has a very high correlation coefficient, and it can be seen from its envelope spectrum that it contains sufficient frequency components. Meanwhile, its kurtosis value also reflects that the component may contain fault pulse information. Although component 1 has the highest kurtosis value, its correlation coefficient is too small, which may be the noise interference component. By removing component 1, some interference components are also removed correspondingly, which is also the purpose of the first layer of filtering.

Since the first layer of reconstruction filter analysis did not obtain very effective fault information, multilayer reconstruction filter analysis can be performed. After analyzing the one-to-three-layer filtering, it is found that the multilayer filter in this experiment is a certain improvement, but the most basic two-layer reconstruction filter can be used for accurate fault diagnosis, which will be described in detail below.

The filter reconstruction signal is input to the output layer. Figure 16 shows the envelope spectrum of the components obtained, and the corresponding kurtosis of each component is given in Table 5. In component 5, there is an extremely prominent fault characteristic frequency component; that is, the characteristic frequency of the outer ring fault is $230.7 \mathrm{~Hz}$. At the same time, there is a relatively less obvious component of double frequency. At the same time, the kurtosis value of component 5 is 4.18 , much larger than 3, which can also reflect that the bearing may have a fault impact. Figure 17 shows the envelope spectrum diagram of the 7050th minute T3 point in Figure 11. At this point, it can be clearly seen that the bearing has an obvious outer ring fault, and the fault characteristic frequency is $230.7 \mathrm{~Hz}$, which is exactly the same as the fault characteristic frequency obtained by the method proposed in this paper after data 


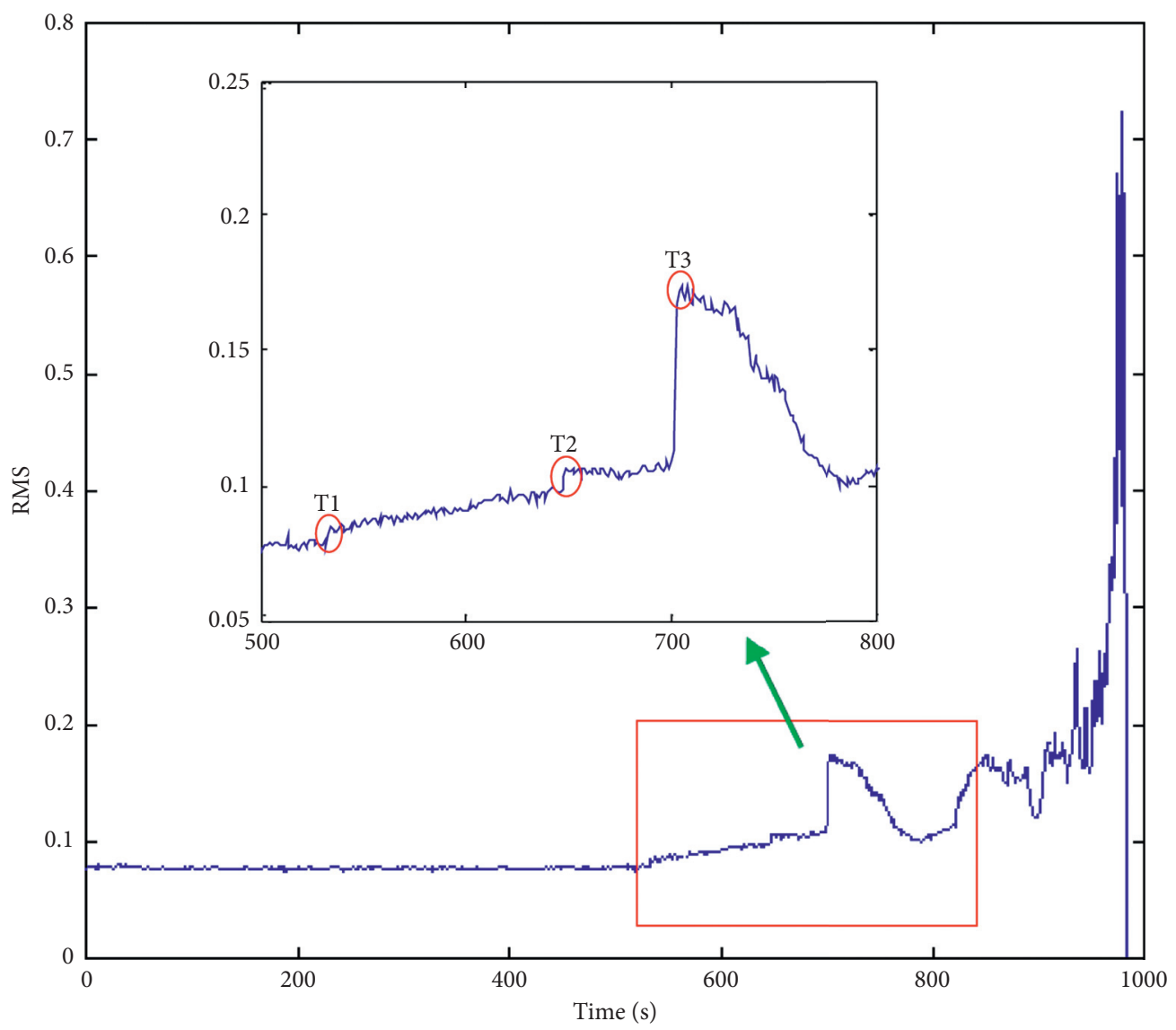

FIgURE 11: Root mean square value diagram of bearing life cycle.

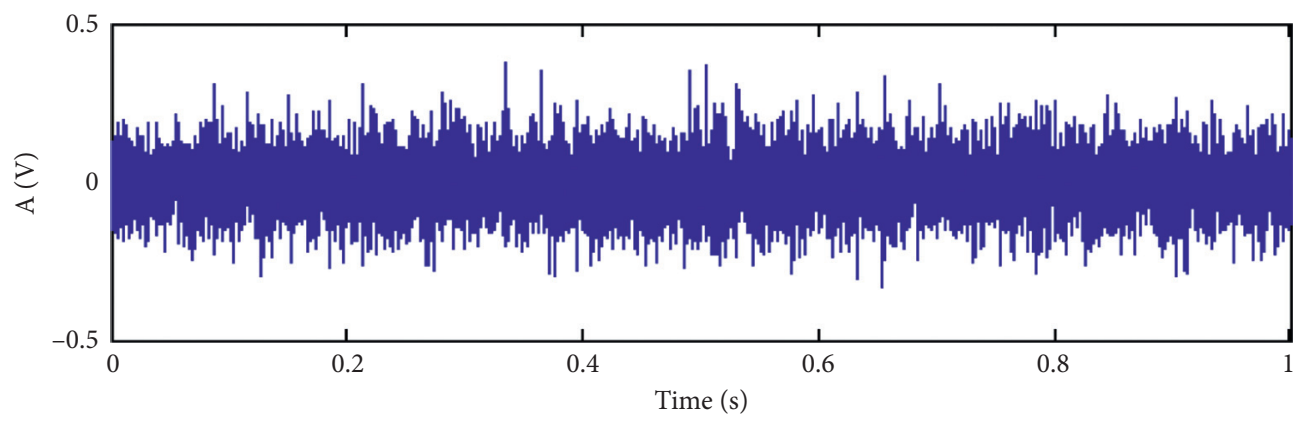

FIGURE 12: Data time domain waveform of the 4520th minute.

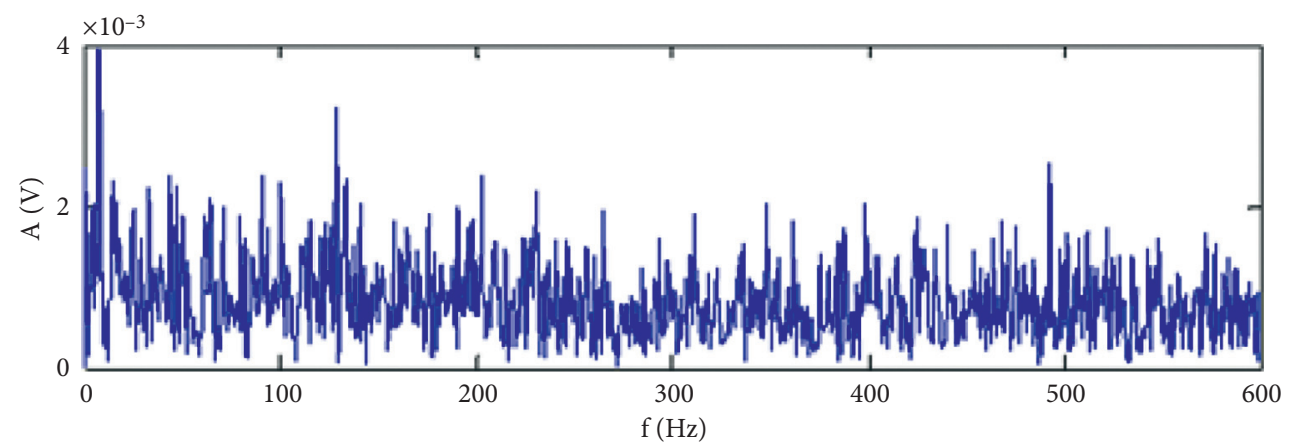

FIgURE 13: The data envelope spectrum at the 4520th minute. 


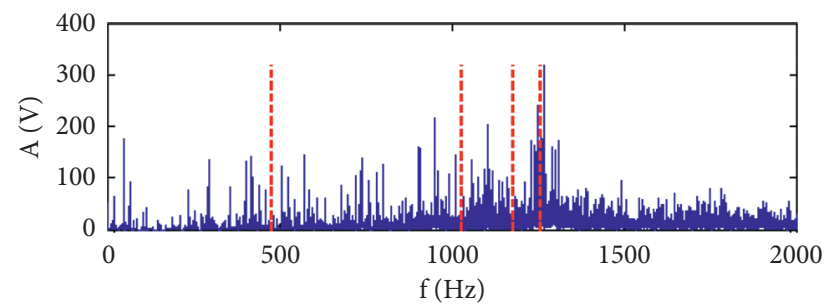

FIgURE 14: EWT boundary detection diagram of experiment 2.
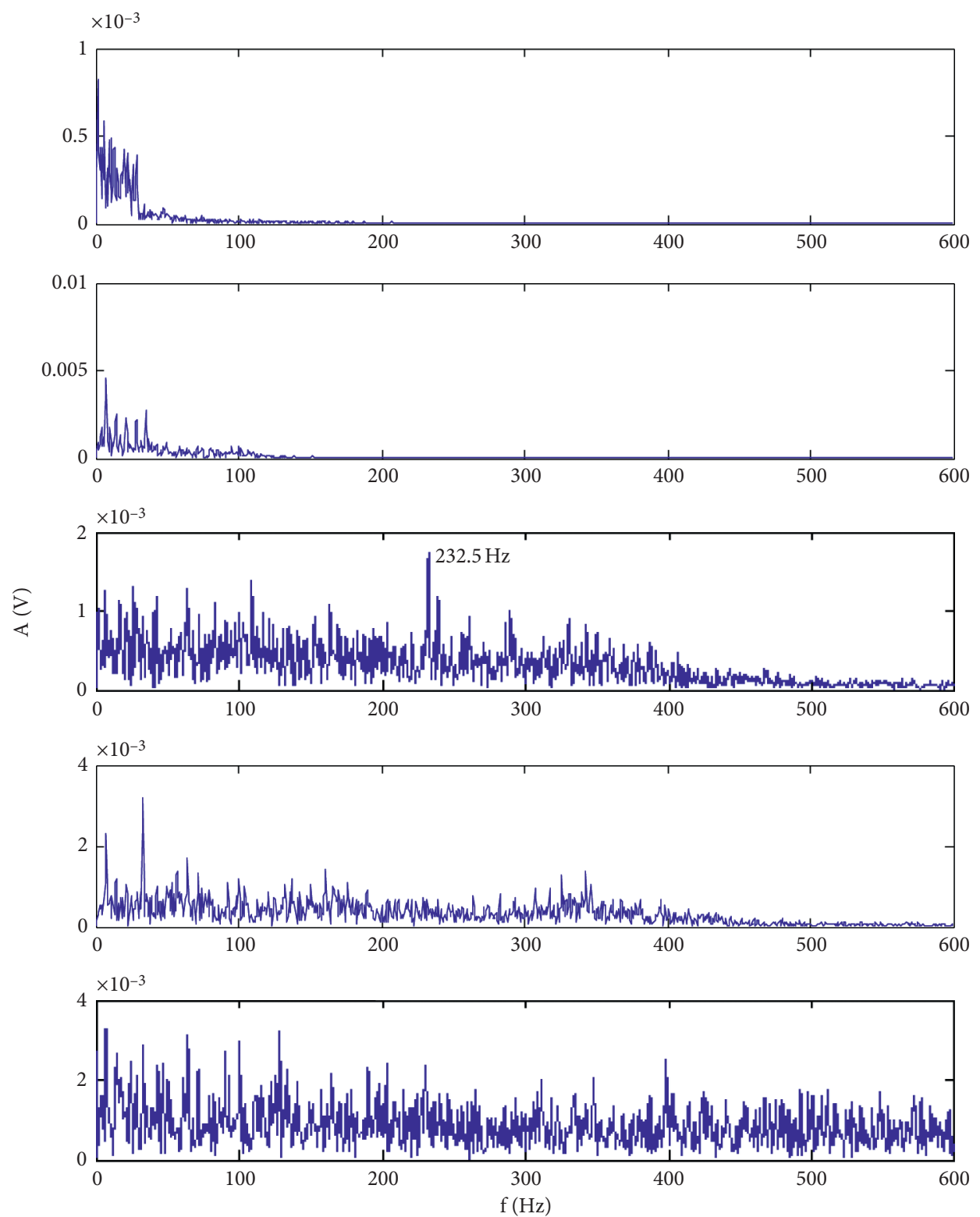

FigURE 15: EWT component envelope spectrum of experiment 2. 
TABLE 4: Correlation coefficient and kurtosis of EWT component of experiment 2.

\begin{tabular}{lll}
\hline & Correlation coefficient & Kurtosis \\
\hline Component 1 & 0.06 & 4.60 \\
Component 2 & 0.20 & 2.92 \\
Component 3 & 0.23 & 2.98 \\
Component 4 & 0.45 & 2.22 \\
Component 5 & 0.94 & 3.55 \\
\hline
\end{tabular}
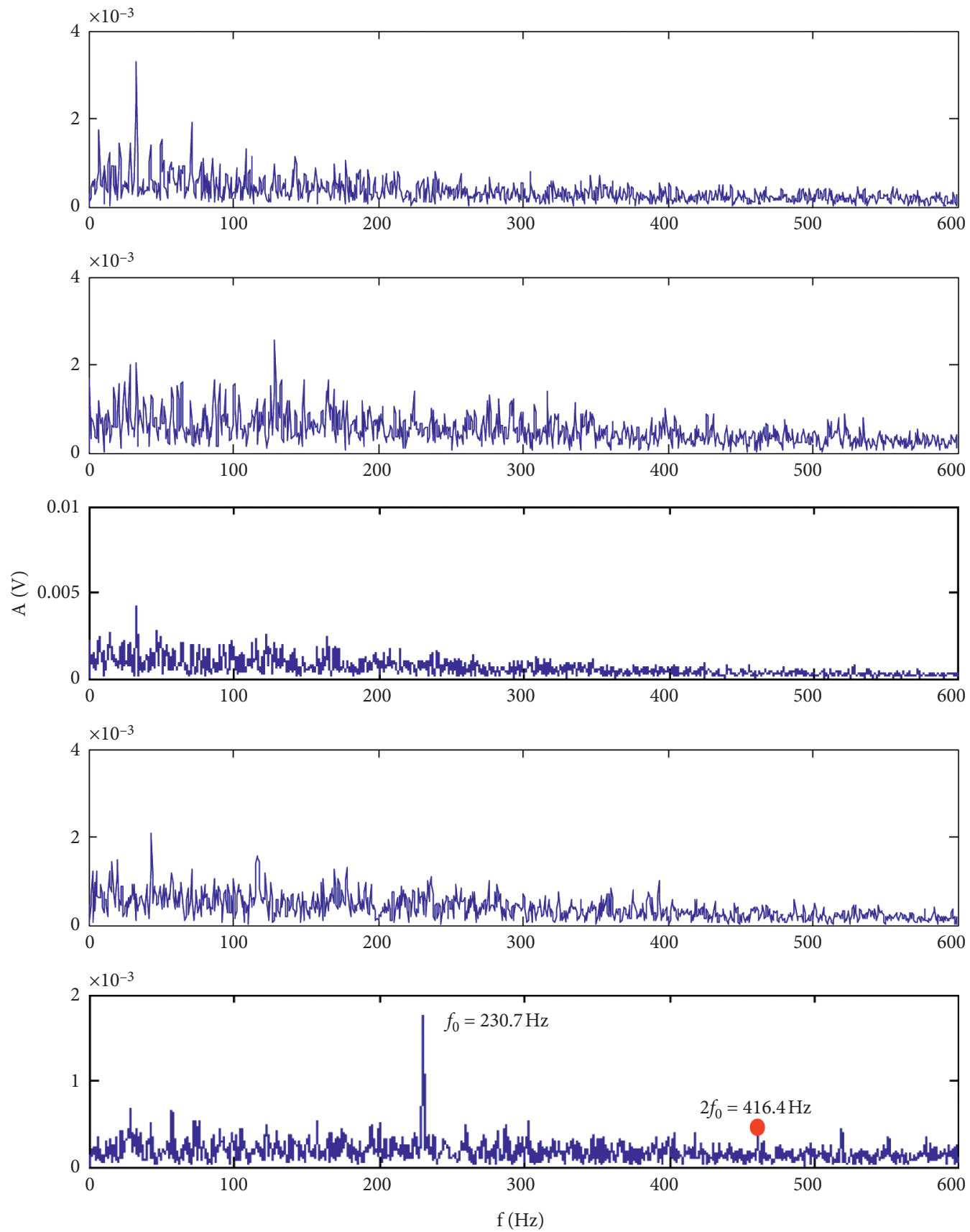

FIGURE 16: VMD component envelope spectrum of experiment 2. 
TABLE 5: Correlation coefficient and kurtosis of EWT component of experiment 2.

\begin{tabular}{lccccc}
\hline & Component 1 & Component 2 & Component 3 & Component 4 & Component 5 \\
\hline Kurtosis & 2.24 & 3.94 & 3.54 & 3.20 & 4.18 \\
\hline
\end{tabular}

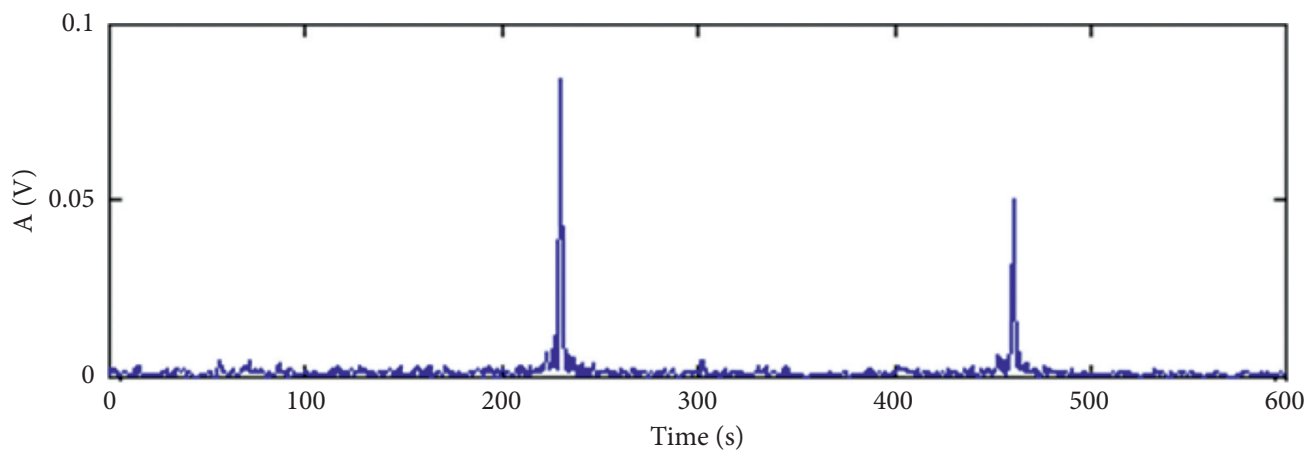

FIgURE 17: The data envelope spectrum at the 7050th minute.

processing of the 4520th minute. Based on the above information, it can be concluded that the bearing has had an early weak failure of the outer ring.

At the same time, we also analyzed the data at other time points. The data after 4520 minutes can effectively identify the fault features, so we will not make a detailed introduction here.

\section{Conclusions}

Aiming at the problem that the early weak fault characteristics of rolling bearing are weak, which is difficult to diagnose in time, this paper proposes a method for early weak fault diagnosis of rolling bearing based on multilayer reconstruction filter, which can effectively extract the fault characteristics from the acoustic and vibration signals, detect the fault earlier, and avoid serious consequences.

The early weak fault diagnosis method of rolling bearing based on multilayer reconstruction filter is proposed, which combines the characteristics of two advanced time-frequency analysis methods EWT and VMD, to gradually remove the interference information, highlight the fault pulse information, extract the early weak fault features, and accurately diagnose the fault. The proposed multilayer reconstruction filter method can be used to decompose the measured signal layer by layer to detect weaker fault characteristics, which means that the rolling bearing fault can be found earlier in the practical application, which is of great significance to the safe operation of the equipment.

In this paper, the validity of the proposed method is verified by the rolling bearing fault experiment and the full life cycle acceleration experiment data. Experiment results show that the proposed method can overcome the challenges brought about by low SNR and weak fault features, extract fault feature information earlier, and avoid the further evolution of the fault, resulting in incalculable losses. At the end of the paper, the future research work is prospected. In the proposed method, the parameters of some steps should be obtained by preprocessing the signal. Therefore, other parameter optimization methods can be integrated into the future work, so that the whole method can be adaptively selected to complete the early fault diagnosis.

\section{Data Availability}

The experimental data used in this study can be obtained from the corresponding author upon request.

\section{Conflicts of Interest}

The authors declare no conflicts of interest.

\section{References}

[1] G. Tang, Y. Wang, Y. Huang, N. Liu, and J. He, "Compound bearing fault detection under varying speed conditions with virtual multichannel signals in Angle domain," IEEE Transactions on Instrumentation and Measurement, vol. 69, no. 8, pp. 5535-5545, 2020.

[2] J. Fan and Z. C. Zhu, "An improved VMD with empirical mode decomposition and its application in incipient fault detection of rolling bearing," IEEE Access, vol. 6, no. 1, pp. 44483-44493, 2018.

[3] C. Qin, D. Wang, Z. Xu, and G. Tang, "Improved empirical wavelet transform for compound weak bearing fault diagnosis with acoustic signals," Applied Sciences, vol. 10, no. 2, pp. 682-692, 2020.

[4] J. J. Li, "Research and application of the fault diagnosis of rolling bearing based on the sound signal," M.S. thesis, Tiedao University, Shijiazhuang, China, 2017.

[5] A. Glowacz, "Fault diagnosis of single-phase induction motor based on acoustic signals," Mechanical Systems and Signal Processing, vol. 117, no. 1, pp. 65-80, 2019.

[6] A. Glowacz, W. Glowacz, Z. Glowacz et al., "Early fault diagnosis of bearing and stator faults of the single-phase induction motor using acoustic signals," Measurement, vol. 69, no. 8, pp. 5535-5545, 2018.

[7] A. Parey and A. Singh, "Gearbox fault diagnosis using acoustic signals, continuous wavelet transform and adaptive neurofuzzy inference system," Applied Acoustics, vol. 147, no. 1, pp. 133-140, 2019. 
[8] D. C. Zhang and E. Stewart, "Intelligent acoustic-based fault diagnosis of roller bearings using a deep graph convolutional network," Measurement, vol. 156, no. 1, 2020.

[9] X. W. Liu and D. L. Pei, "Acoustic signal based fault detection on belt conveyor idlers using machine learning," Advanced Powder Technology, vol. 31, no. 7, 2020.

[10] G. Tang, Y. J. Huang, and Y. T. Wang, "Fractional frequency band entropy for bearing fault diagnosis under varying speed conditions," Measurement, vol. 171, no. 1, 2021.

[11] G. Tang, Y. Wang, Y. Huang, and H. Wang, "Multiple timefrequency curve classification for tacho-less and resamplingless compound bearing fault detection under time-varying speed conditions," IEEE Sensors Journal, vol. 21, no. 4, p. 1, 2020.

[12] Y. Lei, J. Lin, Z. He, and M. J. Zuo, “A review on empirical mode decomposition in fault diagnosis of rotating machinery," Mechanical Systems and Signal Processing, vol. 35, no. 12, pp. 108-126, 2013.

[13] S. Chen, Y. Yang, Z. Peng, S. Wang, W. Zhang, and X. Chen, "Detection of rub-impact fault for rotor-stator systems: a novel method based on adaptive chirp mode decomposition," Journal of Sound and Vibration, vol. 440, no. 1, pp. 83-99, 2019.

[14] S. Q. Wang, C. Y. Wang, Q. Sun, C. Y. Gao, and S. G. Yang, "Radar emitter signal intra-pulse feature extraction based on empirical mode decomposition," Procedia Computer Science, vol. 154, no. 1, pp. 504-507, 2019.

[15] S. D. C. Kiat and E. Y. K. Ng, "A computational intelligence tool for the detection of hypertension using empirical mode decomposition," Computers in Biology and Medicine, vol. 118, 2020.

[16] S. Chen, Y. Yang, Z. Peng, X. Dong, W. Zhang, and G. Meng, "Adaptive chirp mode pursuit: algorithm and applications," Mechanical Systems and Signal Processing, vol. 116, no. 1, pp. 566-584, 2019.

[17] D. Wang, K.-L. Tsui, and Y. Qin, "Optimization of segmentation fragments in empirical wavelet transform and its applications to extracting industrial bearing fault features," Measurement, vol. 133, no. 1, pp. 328-340, 2019.

[18] T. Gong, X. Yuan, X. Lei, and X. Wang, "Application of tentative variational mode decomposition in fault feature detection of rolling element bearing," Measurement, vol. 135, no. 1, pp. 481-492, 2019.

[19] K. Mourad and M. Thomas, "A comparative study between empirical wavelet transforms and empirical mode decomposition methods: application to bearing defect diagnosis," Mechanical Systems and Signal Processing, vol. 81, no. 1, pp. 88-107, 2016.

[20] A. Bhattacharyya and R. B. Pachori, "A multivariate approach for patient-specific EEG seizure detection using empirical wavelet transform," IEEE Transactions on Biomedical Engineering, vol. 64, no. 9, pp. 2003-2015, 2017.

[21] W. Liu, S. Cao, and Y. Chen, "Seismic time-frequency analysis via empirical wavelet transform," IEEE Geoscience and Remote Sensing Letters, vol. 13, no. 1, pp. 28-32, 2016.

[22] J. Hu and J. Wang, "Short-term wind speed prediction using empirical wavelet transform and Gaussian process regression," Energy, vol. 93, no. 1, pp. 1456-1466, 2015.

[23] J. L. Chen and J. Pan, "Generator bearing fault diagnosis for wind turbine via empirical wavelet transform using measured vibration signals," Renewable Energy, vol. 89, no. 89, pp. 80-92, 2020.

[24] J. Pan, J. Chen, Y. Zi, Y. Li, and Z. He, "Mono-component feature extraction for mechanical fault diagnosis using modified empirical wavelet transform via data-driven adaptive Fourier spectrum segment," Mechanical Systems and Signal Processing, vol. 72-73, no. 1, pp. 160-183, 2016.

[25] S. Chen, X. Dong, Z. Peng, W. Zhang, and G. Meng, "Nonlinear chirp mode decomposition: a variational method," IEEE Transactions on Signal Processing, vol. 65, no. 22, pp. 6024-6037, 2017.

[26] G. Li, G. Tang, G. Luo, and H. Wang, "Underdetermined blind separation of bearing faults in hyperplane space with variational mode decomposition," Mechanical Systems and Signal Processing, vol. 120, no. 1, pp. 83-97, 2019.

[27] X. Jiang, J. Wang, J. Shi, C. Shen, W. Huang, and Z. Zhu, “A coarse-to-fine decomposing strategy of VMD for extraction of weak repetitive transients in fault diagnosis of rotating machines," Mechanical Systems and Signal Processing, vol. 116, no. 1, pp. 668-692, 2019.

[28] X. B. Wang and Z. X. Yang, "Novel particle swarm optimization-based variational mode decomposition method for the fault diagnosis of complex rotating machinery," IEEE-ASME Transactions on Mechatronics, vol. 23, no. 1, pp. 68-79, 2018.

[29] Z. Wang, G. He, W. Du et al., "Application of parameter optimized variational mode decomposition method in fault diagnosis of gearbox," IEEE Access, vol. 7, no. 1, pp. 44871-44882, 2019.

[30] Z. Xu, C. Qin, and G. Tang, “A novel deconvolution cascaded variational mode decomposition for weak bearing fault detection with unknown signal transmission path," IEEE Sensors Journal, vol. 21, no. 2, p. 1746, 2021.

[31] H. D. Wang, S. E. Deng, J. X. Yang et al., "Incipient fault diagnosis of rolling bearing based on VMD with parameters optimized," Journal of Vibration and Shock, vol. 39, no. 23, pp. $38-46,2020$. 